\title{
A New Symmetric p-stable Obrechkoff Method with Optimal Phase-lag for Oscillatory Problems
}

\author{
I. C. Felix ${ }^{1, *}$, O. O. Famoofo ${ }^{2}$ and S. M. Akintewe ${ }^{3}$ \\ ${ }^{1,2}$ School of Engineering and Applied Sciences, Lagos City Polytechnic, Ikeja, Lagos State, Nigeria; \\ e-mail: isaac.c.felix@aims-senegal.org ${ }^{1}$; emiaserere@gmail.com ${ }^{2}$ \\ ${ }^{3}$ Department of Mathematical Science, Adekunle Ajasin University, Akungba-Akoko, Ondo State, \\ Nigeria; e-mail: mercy.s.akintewe@gmail.com ${ }^{3}$ \\ * Corresponding author
}

\begin{abstract}
In this paper, we derive a class of symmetric p-stable Obrechkoff methods via Padé approximation approach (PAA) for the numerical solution of special second order initial value problems (IVPs) in ordinary differential equations (ODEs). We investigate periodicity analysis on the proposed scheme to verify p-stability property. The new algorithms possess minimum phase-lag error which shows that they can accurately solve oscillatory problems. Reports on several numerical experiments are provided to illustrate the accuracy of the method.
\end{abstract}

\section{Introduction}

Our task in this paper is to approximate the solution of the special second order IVPs of the form

$$
y^{\prime \prime}=f(x, y), \quad y\left(x_{0}\right)=y_{0}, \quad y^{\prime}\left(x_{0}\right)=y_{0}^{\prime},
$$

Received: January 4, 2019; Accepted: January 18, 2019

2010 Mathematics Subject Classification: 65L05, 65L06.

Keywords and phrases: p-stability, Obrechkoff method, Padé approximation, symmetric, oscillatory, phaselag error.

Copyright (๑ 2019 I. C. Felix, O. O. Famoofo and S. M. Akintewe. This is an open access article distributed under the Creative Commons Attribution License, which permits unrestricted use, distribution, and reproduction in any medium, provided the original work is properly cited. 
where $y(x) \in \mathbb{R}, f: \mathbb{R} \times \mathbb{R}^{g} \rightarrow \mathbb{R}^{g}$, and the first derivative does not appear explicitly. Such problems are often encountered in applied science and engineering. Popular examples include: mechanical systems without dissipation, satellite tracking, celestial mechanics, etc. The solution of the type (1) which is considered in this paper is a priori known to be periodic, and when integrated numerically, the desire is that the numerical solution also preserves the analogical periodicity of the analytic solution [10-23]. Furthermore, equation (1) is known to have inherent "periodic stiffness" [12] which makes it difficult to solve analytically. Numerical methods must be employed to obtain its approximate solution. The well-known Stomer-Cowell method with step number greater than two exhibits orbital instability making it unsuitable to numerically solve (1). Unlike other stability requirements, 2-step p-stable methods remain desirable for the solution of (1). There is vast literature on approximate solution of (1), see [7], [8], [9], [10], [11], [31], [32], [3-5], [2], [24-27], [6], [11], [32], [28-30], [10], [35-37], [20], [33]. The significance of this present work is five-fold; (i) to illustrate the strength of PAA in the development of numerical methods capable of handling IVPs that are periodic in nature, (ii) derive new p-stable methods and investigate their phase-lag properties, (iii) provide useful insight on p-stable Obrechkoff methods, (iv) remark that "direct application of PAA framework" on the development of p-stable numerical methods will often be limited to schemes of order $p \leq 4$, (v) wide application of the derived methods. In what follows, we have demonstrated the accuracy of our methods using stiff, linear and non-linear IVPs in ODEs.

Consider the Obrechkoff method of the form

$$
\sum_{i=0}^{k} \alpha_{i} y_{n-j+1}=\sum_{i=1}^{l} h^{2 i} \sum_{j=0}^{k} \beta_{i j} y_{n-j+1}^{(2 i)},
$$

for the numerical solution of the problem (1). When the method (2) is applied on test equation (3)

$$
y^{\prime \prime}+\lambda^{2} y=0, \quad \lambda, y \in \mathbb{R}
$$

we get the characteristics equation as

$$
\rho(\xi)=\sum_{i=1}^{l}(-1)^{i} v^{2 i} \sigma_{i}(\xi),
$$


where $v-\lambda h=0$ and

$$
\rho(\xi)=\sum_{j=0}^{k} \alpha_{j} \xi^{k-j}, \quad \sigma_{i}(\xi)=\sum_{j=0}^{k} \beta_{i j} \xi^{k-j}, \quad i=1,2, \ldots, l .
$$

Definition 1.1. The method in (2) is said to be symmetric if $\alpha_{j}=\alpha_{k-j}$, $\beta_{j}=\beta_{k-j}, j=0,1,2, \ldots, k$.

Definition 1.2. The method in (2) is said to have order $p$ if the truncation error associated with the linear difference operator is given as

$$
T E=C_{p+2} h^{p+2} y(p+2), \quad x_{n-k+1}<\eta<x_{n+1},
$$

where $C_{p+2}$ is the error constant dependent on $h$.

Definition 1.3. The method in (2) is said to have interval of periodicity $\left(0, v_{0}^{2}\right)$ if for all $v_{0}^{2} \in\left(0, v_{0}^{2}\right)$ the roots of (4) are complex and at least two of them lie on the unit circle and others lie inside the unit circle.

Definition 1.4. The method in (2) is said to be $p$-stable if its interval of periodicity is $(0, \infty)$.

\section{Derivation of the Method}

In the spirit of [10], we consider the following algebraic expressions

$$
\Pi(\xi, z)=\left(P_{j}(z) P_{j}(-z)\right) \xi^{2}-\left(P_{j}^{2}(z)+P_{j}^{2}(-z)\right) \xi+\left(P_{j}(z) P_{j}(-z)\right)
$$

with $z \in \mathbb{C}$ and $P_{j}$ described by the expression

$$
P_{j}(z)=1+\frac{j}{2 j} z+\frac{j(j-1)}{(2 j)(2 j-1)} \frac{z^{2}}{2 !}+\cdots+\frac{j(j-1) \cdots 2 \cdot 1}{(2 j)(2 j-1) \cdots(j+1)} \frac{z^{j}}{j !}
$$

whose roots is given by

$$
\xi_{1}=\left(\xi_{2}\right)^{-1}=\frac{P_{j}(z)}{P_{j}(-z)},
$$

such that

$$
\left\|R_{j, j}(z)-e^{z}\right\| \leq O\left(\|z\|^{2 j+1}\right), \quad z \rightarrow 0
$$


is known as the $(j, j)$-diagonal Padé approximation to $e^{z}$. Observe that (7) is of the form (4). In what follows, the basic idea is to obtain the stability function of an integration scheme and compare it with (7) to determine the corresponding coefficients of the numerical method.

Consider the symmetric Obrechkoff methods (2), when applied on (3) yield the following stability function

$$
\begin{aligned}
\Pi(\xi, z)= & \left(\alpha_{2}-z^{2} \beta_{10}-z^{4} \beta_{20}\right) \xi^{2}+\left(\alpha_{1}-z^{2} \beta_{11}-z^{4} \beta_{21}\right) \xi \\
& +\left(\alpha_{2}-z^{2} \beta_{10}-z^{4} \beta_{20}\right) .
\end{aligned}
$$

Without loss of generality, we compare (11) with results from which $j=2$ in (7) and obtain the following symmetric p-stable Obrechkoff method

$$
y_{n+1}-2 y_{n}+y_{n-1}=\frac{h^{2}}{12 \gamma}\left[10 y_{n}^{\prime \prime}+\left(y_{n+1}^{\prime \prime}+y_{n-1}^{\prime \prime}\right)\right]+\frac{h^{4}}{72 \gamma}\left[y_{n}^{\prime \prime}-\frac{1}{2}\left(y_{n+1}^{\prime \prime}+y_{n-1}^{\prime \prime}\right)\right],
$$

where $\gamma \geq 10^{3}$. Figure 1 shows an indefinite progression of the new method along the positive real axis satisfying Definition 1.4 ,

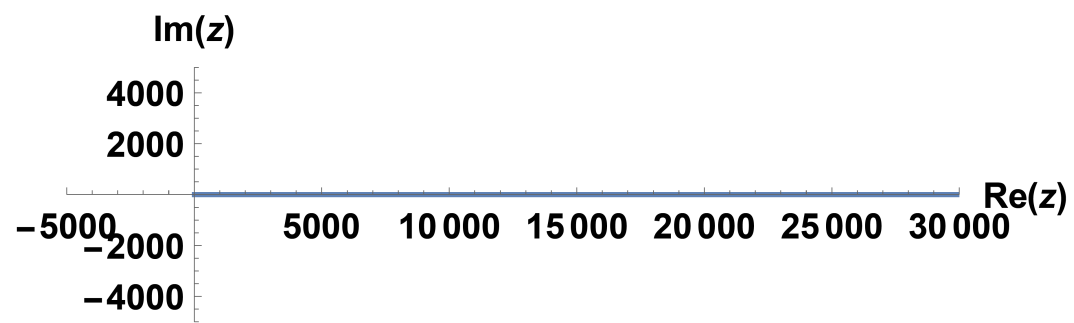

Figure 1. The stability plot of scheme (12).

Remarkably, the stability region of the famed most accurate p-stable formulas satisfying Definition 1.4 is given in Figure 2

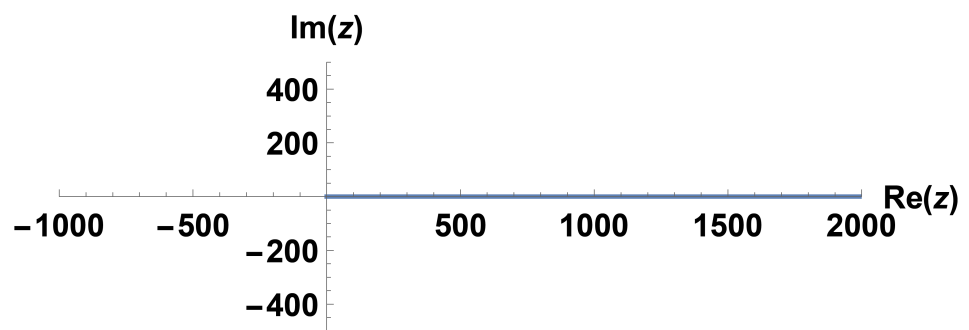

Figure 2. The stability plot of the famed most accurate p-stable method. 
Also, in [10] the following methods are proposed

$$
\begin{gathered}
\sum_{j=0}^{k} \alpha_{j} y_{n+j}=h^{2} \sum_{j=0}^{k} \beta_{j} f\left(t_{n+j}, y_{n+j}\right)+h^{2} \beta f\left(\tilde{t}_{n}, \tilde{y}_{n}\right) \\
\tilde{y}_{n}=\sum_{j=0}^{k} \gamma_{j} y_{n+j}+h^{2} \sum_{j=0}^{k} \delta_{j} f\left(t_{n+j}, y_{n+j}\right),
\end{gathered}
$$

where $h$ is the step size and

$$
t_{j}=t_{0}+j h, \quad \tilde{t}_{n}=t_{n}+\left(\sum_{j=0}^{k} \gamma_{j} j\right) h .
$$

When applied to the scalar test (3) yields a difference equation with the following characteristics polynomial

$$
\sum_{j=0}^{k}\left[\alpha_{j}-z^{2}\left(\beta_{j}+\beta \gamma_{j}\right)-z^{4} \beta \delta_{j}\right] R^{j}=0 .
$$

The interval of periodicity of this scheme is found to be indeed p-stable. Figure 3 shows the stability region of the integration scheme. Clearly, this grows indefinitely along the positive real axis with however some insignificant jumps at the origin which could be as a result of parameter choice.

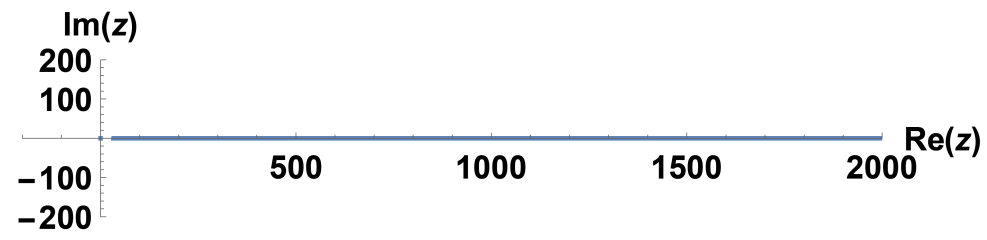

Figure 3. Stability plot showing the interval of periodicity of scheme (13).

Next, we carry out phase-lag analysis on the method (12) following [20-22], see also [27-29] and [31-33]. The phase-lag analysis of numerical method is described by

$$
P L(H)=\frac{2 \sum_{j=1}^{\frac{k^{\prime}}{2}} A_{j}\left(H^{2}\right) \cos (j H)+A_{0}\left(H^{2}\right)}{2 \sum_{j=1}^{\frac{k^{\prime}}{2}} j^{2} A_{j}\left(H^{2}\right)}
$$


and when expanded has a non-vanishing phase-lag order $d$ and phase-lag constant $c_{d+2}$.

$$
\begin{aligned}
P L(H) & =\frac{2 A_{\frac{k^{\prime}}{2}}\left(H^{2}\right) \cos \left(\frac{k^{\prime}}{2} H\right)+\cdots+2 A_{j}\left(H^{2}\right) \cos (j H)+A_{0}\left(H^{2}\right)}{2\left(\frac{k^{\prime}}{2}\right)^{2}+\cdots+2 j^{2} A_{j}\left(H^{2}\right)+\cdots+2 A_{1}\left(H^{2}\right)} \\
& =-c_{d+2} H^{d+2}+O\left(H^{d+4}\right) .
\end{aligned}
$$

In what follows, the application of (18) on the derived method (12) yields the following phase-lag

$$
-\frac{1}{120} z^{6}+0\left(z^{8}\right)=-c_{d+2} z^{d+2}+0\left(z^{d+4}\right)
$$

where $-c_{d+2}$ is the phase-lag error (PLE) constant, and $d$, the phase-lag order.

Remark. For all values of $j$ in (7), p-stable numerical methods obtained by directly applying PAA will be limited to order of accuracy $p \leq 4$.

\section{Numerical Experiment and Results}

In this section, we carry out numerical experiments in order to show the accuracy of the new algorithm. Consider the following second order IVPs.

Example 1. (An orbital problem): Source [12]

$$
\begin{gathered}
y^{\prime \prime}=-y+0.001 e^{i x} \\
y(0)=1, \quad y^{\prime}(0)=0.9995 i, \quad i^{2}=-1
\end{gathered}
$$

and has a theoretical solution

$$
\begin{gathered}
y(x)=u(x)+v(x) \\
u(x)=\cos x+0.0005 x \sin x \\
v(x)=\sin x-0.0005 x \cos x .
\end{gathered}
$$

The initial value problem (20) represents a motion on a perturbed circular orbits in the complex plane in which the point $y(x)$ slowly spirals outward such that its distance from the origin at $a$ any given time $x$ is described by 


$$
\Psi(x)=\sqrt{u^{2}(x)+v^{2}(x)} .
$$

Using the predictor

$$
y_{n+2}-2 y_{n+1}+y_{n}=h^{2} f_{n+1},
$$

the system of equation in (20) can be approximated in the interval $[0,40]$ which corresponds to 20 orbits of the points $y(x)$. The integration is then carried out with uniform meshsizes

$$
h=\frac{\pi}{2^{q}}, \quad q=3(1) 10 .
$$

The numerical results from the new p-stable Obrechkoff methods comparing with existing p-stable numerical methods from the literature are presented in Table 1 and Table 2 respectively.

Table 1. $\Psi\left(x_{f}\right)=1.00197197653449$.

\begin{tabular}{|c|c|c|c|}
\hline \hline$q$ & $h$ & New symmetric Obrechkoff method (12)( $\Psi)$ & Error \\
\hline 3 & $\frac{\pi}{2^{3}}$ & 1.001848523273178 & $1.24 \mathrm{E}-4$ \\
\hline 4 & $\frac{\pi}{2^{4}}$ & 1.001801747000679 & $1.70 \mathrm{E}-4$ \\
\hline 5 & $\frac{\pi}{2^{5}}$ & 1.001987397382566 & $1.54 \mathrm{E}-5$ \\
\hline 6 & $\frac{\pi}{2^{6}}$ & 1.001974909077729 & $2.93 \mathrm{E}-6$ \\
\hline 7 & $\frac{\pi}{2^{7}}$ & 1.001973299992589 & $1.32 \mathrm{E}-6$ \\
\hline 8 & $\frac{\pi}{2^{8}}$ & 1.001972374396628 & $3.98 \mathrm{E}-7$ \\
\hline 9 & $\frac{\pi}{2^{9}}$ & 1.001972355120986 & $3.79 \mathrm{E}-7$ \\
\hline 10 & $\frac{\pi}{2^{10}}$ & 1.001972345485908 & $3.69 \mathrm{E}-7$ \\
\hline
\end{tabular}


Table 2. Continuation of Table 1.

\begin{tabular}{|c|c|c|c|c|c|c|c|}
\hline \hline$q$ & $h$ & $\begin{array}{c}\text { Method in } \\
{[12]}\end{array}$ & Error & $\begin{array}{c}\text { Method in } \\
{[10]}\end{array}$ & Error & $\begin{array}{c}\text { Method in } \\
{[2]}\end{array}$ & Error \\
\hline 3 & $\frac{\pi}{2^{3}}$ & 0.965645 & $3.63 \mathrm{E}-2$ & 0.994863 & $7.11 \mathrm{E}-3$ & NA & NA \\
\hline 4 & $\frac{\pi}{2^{4}}$ & 0.993734 & $8.23 \mathrm{E}-3$ & 0.997223 & $4.75 \mathrm{E}-3$ & 1.004118 & $2.15 \mathrm{E}-3$ \\
\hline 5 & $\frac{\pi}{2^{5}}$ & 0.999596 & $2.38 \mathrm{E}-3$ & 0.997578 & $4.39 \mathrm{E}-3$ & 1.002856 & $8.84 \mathrm{E}-4$ \\
\hline 6 & $\frac{\pi}{2^{6}}$ & NA & NA & 0.997687 & $4.39 \mathrm{E}-3$ & 1.002400 & $4.3 \mathrm{E}-4$ \\
\hline 7 & $\frac{\pi}{2^{7}}$ & NA & NA & 0.997730 & $4.24 \mathrm{E}-3$ & NA & NA \\
\hline 8 & $\frac{\pi}{2^{8}}$ & NA & NA & 0.997748 & $4.22 \mathrm{E}-3$ & NA & NA \\
\hline 9 & $\frac{\pi}{2^{9}}$ & 1.001829 & $1.43 \mathrm{E}-4$ & 0.997757 & $4.22 \mathrm{E}-3$ & 1.002057 & $8.50 \mathrm{E}-5$ \\
\hline 10 & $\frac{\pi}{2^{10}}$ & NA & NA & 0.997761 & $4.21 \mathrm{E}-3$ & NA & NA \\
\hline
\end{tabular}

In what follows, we apply the derived method (12) to investigate the orbital property of problem (20),

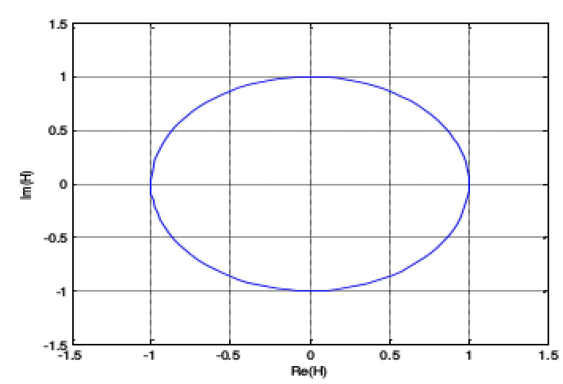

Analytical Solution

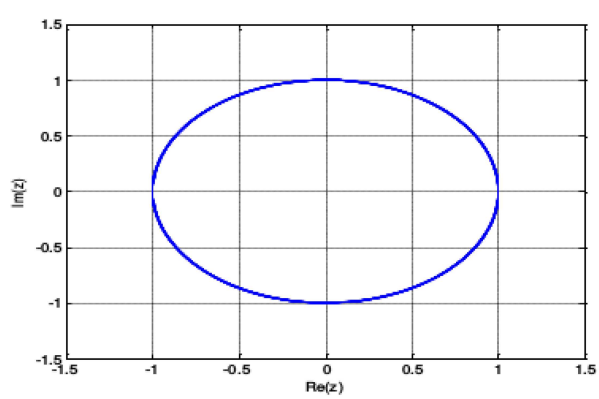

Numerical Solution

Figure 4. Numerical simulation of an orbital problem comparing with its analytical solution. 
Example 2. (Stiff Oscillatory Problem: Source [14])

Consider the stiff oscillatory problem defined by

$$
y^{\prime \prime}(t)+m^{2} y(t)=8\left(\cos (t)+\frac{2}{3} \cos (3 t)\right), \quad y(0)=1, y^{\prime}(0)=0 \quad \text { with } \quad m=5
$$

whose exact solution is

$$
y(t)=\frac{1}{3}(\cos t+\cos 3 t+\cos 5 t)
$$

In similar manner, we implement the system (25) using meshsize $h=\frac{\pi}{8}$ at $t=10 \pi$.

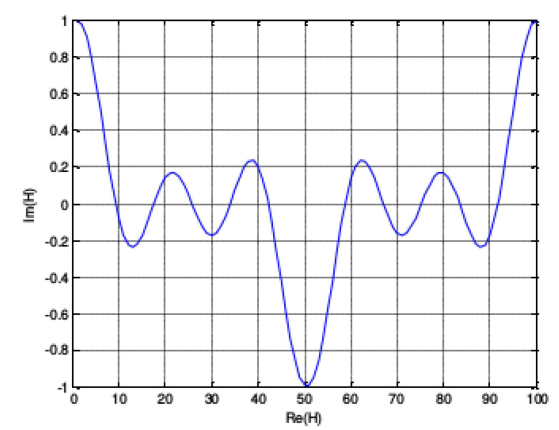

Analytical Solution

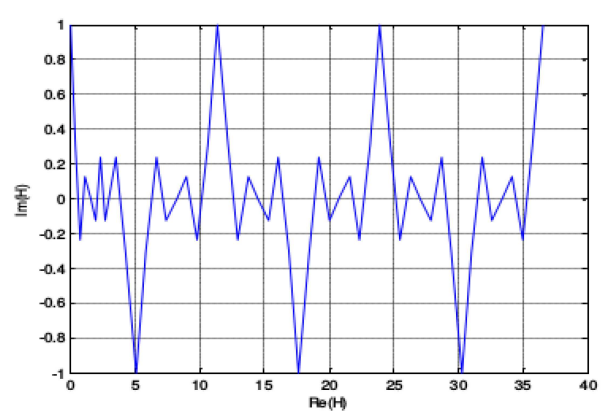

Numerical Solution

Figure 5. Numerical simulation of stiff oscillatory problem comparing with its analytical solution.

Example 3. (Undamped Duffing Problem: Source [3])

Consider again a non-linear duffing problem,

$$
y^{\prime \prime}+y+y^{3}=\delta \cos (\mu t), \quad y(0)=A, \quad y^{\prime}(0)=0 .
$$

The initial condition described by $A$ is the value of the Galerkin's approximation $y_{G}$ at $t=0$. Problem (27) is forced by a harmonic function with parameter values $\delta=0.002$ and $\mu=1.01$. However, by Urabe's method applied to Galerkin's procedure, Van Dooren has carried out computation of the Galerkin's approximation of order $p=9$ to a periodic solution having the same period as the forcing term with a precision of the coefficients of $\frac{1}{10^{12}}$ : 


$$
y_{G}=\sum_{i=0}^{5} \alpha_{2 i+1} \cos (2 i+1) \mu t
$$

in a simplified form, (28) becomes

$$
\begin{aligned}
y_{G}= & \alpha_{1} \cos \mu t+\alpha_{3} \cos (3 \mu t)+\alpha_{5} \cos (5 \mu t)+\alpha_{7} \cos (7 \mu t) \\
& +\alpha_{9} \cos (9 \mu t)+\alpha_{11} \cos (11 \mu t),
\end{aligned}
$$

where

$$
\begin{aligned}
& \alpha_{1}=0.200179477536, \alpha_{3}=0.246946143 \times 10^{-3}, \alpha_{5}=0.304014 \times 10^{-6} \\
& \alpha_{7}=0.374 \times 10^{-9}, \alpha_{9}=0.460964452 \times 10^{-12}, \alpha_{11}=0.5676 \times 10^{-15}
\end{aligned}
$$

Using step size $h=\frac{\pi}{2^{3}}$ at $t=40 \pi$, we apply the new method on problem (27) and obtained the following behaviours

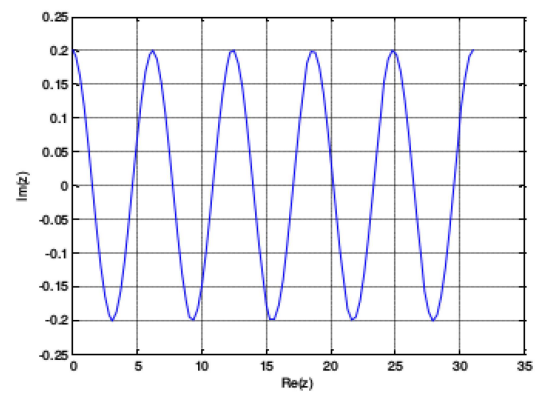

Analytical Solution

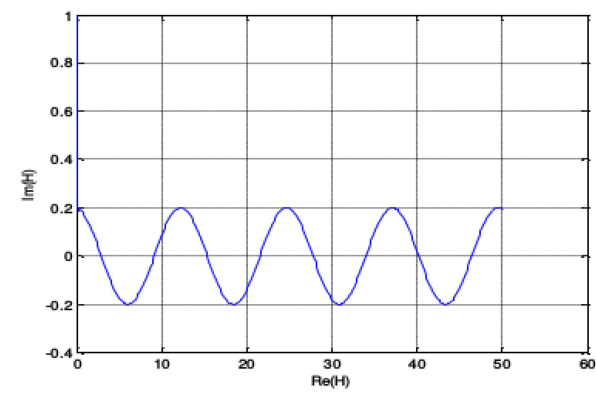

Numerical Solution

Figure 6. Numerical simulation of undamped duffing problem comparing with its analytical solution.

\section{Conclusion}

PAA is an interesting method for the development of numerical schemes that must possess p-stability property. In view of the foregoing, we present a class of symmetric p-stable Obrechkoff methods which possess minimum phase-lag error for the solutions of IVPs that are periodic in nature. However, we desired higher order p-stable method, but due to the limitation encountered in the use of PAA, we therefore remark that numerical algorithms obtained by directly applying PAA will often be limited to order $(p \leq 4)$, see 
also the works in $[7,8,10]$. In particular, the new results in this article enjoy considerable order of accuracy and stronger p-stability property which are illustrated in Table 1 and Figures 1, 4, 5, 6, respectively.

\section{Acknowledgements}

The authors are indebted to Professors M. N. O. Ikhile and R. I. Okuonghae (Department of Mathematics, University of Benin) for their detailed comments and helpful suggestions.

\section{References}

[1] S. D. Achar, Symmetric multistep Obrechkoff methods with zero phase-lag for periodic initial value problems of second order differential equations, J. Appl. Math. Comput. 218 (2011), 2237-2248.

[2] J. R. Cash, High order $P$-stable formulae for the numerical integration of periodic initial value problems, Numer. Math. 37 (1981), 355-370.

[3] M. M. Chawla and B. Neta, Families of two-step fourth order $P$-stable methods for second order differential equations, J. Comput. Appl. Math. 15 (1986), 213-223.

[4] M. M. Chawla and P. S. Rao, A Numerov-type method with minimal phase-lag for the integration of second order periodic initial value problems, J. Comput. Appl. Math. 15 (1986), 329-337.

[5] M. M. Chawla, P. S. Rao and B. Neta, Two-step fourth order P-stable methods with phase-lag of order six for $y=f(t, y)$, J. Comput. Appl. Math. 16 (1986), 233-236.

[6] G. Dahlquist, On accuracy and unconditional stability of linear multistep methods for second order differential equations, BIT 18(2) (1978), 133-136.

[7] S. O. Fatunla, M. N. O. Ikhile and F. O. Otunta, A class of $p$-stable linear multistep numerical methods, Int. J. Comput. Math. 72 (1999), 1-13.

[8] I. C. Felix and R. I. Okuonghae, On the construction of $p$-stable hybrid multistep methods for second order ODEs, Far East J. Appl. Math. 99(3) (2018), 259-273.

[9] T. A. Anake, I. C. Felix and O. P. Ogundile, Higher order super-implicit hybrid multistep methods for second order differential equations, International Journal of Mechanical Engineering and Technology 9(8) (2018), 1384-1392.

[10] I. E. Hairer, Unconditionally stable methods for second order differential equations, Numer. Math. 32 (1979), 373-379. 
[11] M. K. Jain, R. K. Jain and U. A. Krishnaiah, Obrechkoff methods for periodic initial value problems of second order differential equations, J. Math. Phys. Sci. 15 (1981), 239250 .

[12] J. D. Lambert and I. A. Watson, Symmetric multistep methods for periodic initial value problems, J. Inst. Math. Appl. 18 (1976), 189-202.

[13] B. Neta, P-stable high-order super-implicit and Obrechkoff methods for periodic initial value problems, Comput. Math. Appl. 54 (2007), 117-126.

[14] B. Neta, $P$-stable symmetric super-implicit methods for periodic initial value problems, Comput. Math. Appl. 50 (2005), 701-705.

[15] B. Neta, Trajectory propagation using information on periodicity, in: Proc. AIAA/AAS Astrodynamics Specialist Conference, Boston, MA, 10-12 August 1998, Paper Number AIAA 98-4577, 1998.

[16] B. Neta and T. Fukushima, Obrechkoff versus super-implicit methods for the integration of keplerian orbits, in: Proc. AIAA/AAS Astrodynamics Specialist Conference, Denver, CO, 14-17 August 2000, Paper Number AIAA 2000-4029, 2000.

[17] B. Neta and T. Fukushima, Obrechkoff versus super-implicit methods for the solution of first- and second-order initial value problems, Special issue on Numerical Methods in Physics, Chemistry and Engineering, Comput. Math. Appl. 45 (2003), 383-390.

[18] G. Psihoyios and T. E. Simos, Effective numerical approximation of Schrödinger type equations through multiderivative exponentially-fitted schemes, Appl. Numer. Anal. Comput. Math. 1(1) (2004), 205-215.

[19] G. D. Quinlan and S. Tremaine, Symmetric multistep methods for the numerical integration of planetary orbits, Astron. J. 100(5) (1990), 1694-1700.

[20] A. D. Raptis and T. E. Simos, A four-step phase-fitted method for the numerical integration of second order initial value problems, BIT 31 (1991), 160-168.

[21] T. E. Simos, A two-step method with phase-lag of order infinity for the numerical integration of second order periodic initial-value problem, Internat. J. Comput. Math. 39 (1991), 135-140.

[22] T. E. Simos and P. S. Williams, A finite-difference method for the numerical solution of the Schrödinger equation, J. Comput. Appl. Math. 79(2) (1997), 189-205.

[23] D. P. Sakas and T. E. Simos, A family of multiderivative methods for the numerical solution of the Schrödinger equation, J. Math. Chem. 37 (2005), 317-331.

[24] D. P. Sakas and T. E. Simos, Multiderivative methods of eighth algebraic order with 
minimal phase-lag for the numerical solution of the radial Schrödinger equation, $J$. Comput. Appl. Math. 175 (2005), 161-172.

[25] D. P. Sakas and T. E. Simos, Trigonometrically-fitted multiderivative methods for the numerical solution of the radial Schrödinger equation, MATCH Commun. Math. Comput. Chem. 53 (2005), 299-320.

[26] G. Saldanha and S. D. Achar, Symmetric multistep Obrechkoff methods with zero phaselag for periodic initial value problems of second order differential equations, Appl. Math. Comput. 218 (2011), 2237-2248.

[27] A. Shokri, The symmetric two-step $P$-stable nonlinear predictor-corrector methods for the numerical solution of second order initial value problems, Bull. Iranian Math. Soc. 41 (2015), 201-215.

[28] A. Shokri, M. Y. Rahimi Ardabili, S. Shahmorad and G. Hojjati, A new two-step P-stable hybrid Obrechkoff method for the numerical integration of second-order IVPs, J. Comput. Appl. Math. 235 (2011), 1706-1712.

[29] A. Shokri and A. A. Shokri, Implicit one-step L-stable generalized hybrid methods for the numerical solution of first order initial value problems, Iranian J. Math. Chem. 4(2) (2013), 201-212.

[30] A. Shokri and A. A. Shokri, The new class of implicit $L$-stable hybrid Obrechkoff method for the numerical solution of first order initial value problems, Comput. Phys. Commun. 184 (2013), 529-531.

[31] T. E. Simos, A $P$-stable complete in phase Obrechkoff trigonometric fitted method for periodic initial value problems, Proc. Roy. Soc. London Ser. A 441 (1993), 283-289.

[32] T. E. Simos, Exponentially fitted multiderivative methods for the numerical solution of the Schrödinger equation, J. Math. Chem. 36 (2004), 13-27.

[33] T. E. Simos, Multiderivative methods for the numerical solution of the Schrödinger equation, Commun. Math. Comput. Chem. 50 (2004), 7-26.

[34] B. P. Sommeijer, P. J. Van der Houwen and B. Neta, Symmetric linear multistep methods for second order differential equations with periodic solutions, J. Appl. Numer. Math. 2 (1986), 69-77.

[35] E. Steifel and D. G. Bettis, Stabilization of Cowells methods, Numer. Math. 13 (1969), 154-175.

[36] Ch. Tsitouras and T. E. Simos, Explicit high order methods for the numerical integration of periodic initial-value problems, Appl. Math. Comput. 95(1) (1998), 15-26. 
[37] M. Van Daele and G. Vanden Berghe, P-stable exponentially fitted Obrechkoff methods of arbitrary order for second order differential equations, Numer. Algorithms 46 (2007), 333-350.

[38] Z. Wang and Y. Wang, A new kind of high-efficient and high-accurate P-stable Obrechkoff three-step method for periodic initial-value problems, Comput. Phys. Comm. 171(2) (2005), 79-92.

[39] Z. Wang, D. Zhao, Y. Dai and X. Song, A new high efficient and high accurate Obrechkoff four-step method for the periodic non-linear undamped duffings equation, Comput. Phys. Comm. 165 (2005), 110-126.

[40] Z. Wang, D. Zhao, Y. Dai and D. Wu, An improved trigonometrically fitted $P$-stable Obrechkoff method for periodic initial-value problems, Proc. R. Soc. 461 (2005), 16391658. 